\title{
How a haemosporidian parasite of bats gets around: the genetic structure of a parasite, vector and host compared
}

\author{
F. WITSENBURG ${ }^{*}$ L. CLÉMENT ${ }^{*}$ A. LÓPEZ-BAUCELLS,† J. PALMEIRIM,† I. PAVLINIĆ, $\S$

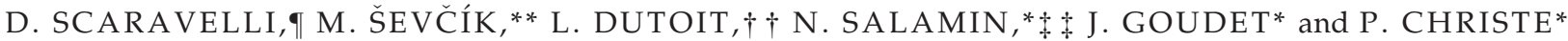 \\ *Department of Ecology and Evolution, University of Lausanne, Biophore, Lausanne CH-1015, Switzerland, †Bat Research Area, \\ Granollers Museum of Natural Sciences, Carrer Palaudaries 102, Granollers 08402, Spain, \$Centre for Environmental Biology, \\ Department of Animal Biology, University of Lisbon, Lisbon 1749-016, Portugal, §Department of Zoology, Croatian Natural \\ History Museum, Demetrova 1, Zagreb HR-10000, Croatia, \Laboratory of Pathogens' Ecology, Department of Veterinary \\ Medical Sciences, University of Bologna, Via Tolara di Sopra 50, Ozzano Emilia (BO) 40064, Italy, **Department of Zoology, \\ Faculty of Science, Charles University in Prague, Viničná 7, Praha 2 CZ-128 44, Czech Republic, ††Department of Evolutionary \\ Biology, Evolutionary Biology Centre, Uppsala University, Norbyvägen 18D, Uppsala SE-752 36, Sweden, \$+Swiss Institute of \\ Bioinformatics, Genopode, Lausanne CH-1015, Switzerland
}

\begin{abstract}
Parasite population structure is often thought to be largely shaped by that of its host. In the case of a parasite with a complex life cycle, two host species, each with their own patterns of demography and migration, spread the parasite. However, the population structure of the parasite is predicted to resemble only that of the most vagile host species. In this study, we tested this prediction in the context of a vector-transmitted parasite. We sampled the haemosporidian parasite Polychromophilus melanipherus across its European range, together with its bat fly vector Nycteribia schmidlii and its host, the bent-winged bat Miniopterus schreibersii. Based on microsatellite analyses, the wingless vector, and not the bat host, was identified as the least structured population and should therefore be considered the most vagile host. Genetic distance matrices were compared for all three species based on a mitochondrial DNA fragment. Both host and vector populations followed an isolation-by-distance pattern across the Mediterranean, but not the parasite. Mantel tests found no correlation between the parasite and either the host or vector populations. We therefore found no support for our hypothesis; the parasite population structure matched neither vector nor host. Instead, we propose a model where the parasite's gene flow is represented by the added effects of host and vector dispersal patterns.
\end{abstract}

Keywords: co-evolution, dispersal, Haemosporida, Nycteribiidae, population genetics, vectortransmitted parasite

Received 30 September 2014; revision received 20 December 2014; accepted 2 January 2015

\section{Introduction}

Parasites are more and more being used as genetic tags of their hosts, which can give additional demographic information when a host population shows low genetic variability (McCoy et al. 2005a; Bruyndonckx et al. 2010). Moreover, parasites can reveal nonreproductive contact between different host populations or even

Correspondence: F. Witsenburg, Fax: +41(0)21 692 4165; E-mail: fardo.witsenburg@unil.ch different host species (Bruyndonckx et al. 2009). Naturally, not all parasite species are reliable tags: their particular mating systems, life-history traits or demographic characteristics can make them less convenient or positively misleading proxies for a host's genealogy (Nieberding \& Olivieri 2007).

Parasites with complex life cycles are parasites which need a minimum of two hosts to complete their life cycle. Such heteroxenous parasites are potentially unsuitable tags as any genetic signal should be a mix of that of the two host species. How exactly the 
population structure of heteroxenous parasites is shaped by both hosts remains unclear. Jarne \& Théron (2001) proposed that, a priori, the population structure of a heteroxenous parasite should closely mimic that of its least structured host. The reasoning being that for its overall gene flow, the parasite is dependent on the host with highest dispersal. Any potential signal of the more strongly isolated species would be erased by a more motile host with more frequent dispersal events (Louhi et al. 2010).

The strength of such correlations between parasite and host population structures are, however, also dependent on their respective life-history traits. For parasites, the duration of the free-living stage in particular could influence the tightness of this correlation. The longer the free-living stage of a parasite lasts, the more incongruence would be expected between parasite and host. Spores and eggs have the potential to be distributed over a large range, yet their effect on the parasites gene flow has been studied only minimally (Jarne \& Théron 2001). Important host traits that could alter the expected relation between parasite and host gene flow include the host's timing of reproduction and dispersal.

Many socially and economically important diseases, such as malignant malaria, chagas and Lyme disease, are caused by vector-transmitted parasites. These can be considered a special class of heteroxenous parasites, for several reasons. First of all, the two host species required for completion of their life cycle are from much diverged taxa, different phyla in general. Moreover, these parasites are obligate, permanent parasites; they cannot live outside the host and have no free-living stages. As a consequence, for the parasite to complete its life cycle, multiple encounters between the host and vector species are needed. A last peculiarity of many vectored parasites is that one of the two hosts, 'the vector', is itself dependent on the other host for survival and can be considered a parasite itself.

Despite the peculiarities of the system, surprisingly, few studies have looked into how vector and host populations interact in shaping the genetic structure of the parasite. Levin \& Parker (2013) found a complete absence of differentiation in an island population of the haemosporidian parasite Haemoproteus iwo. The hippoboscid vector Olfersia spinifera had much lower population differentiation than the avian host Fregata minor, which might explain the lack of differentiation in H. iwo. However, the authors could not compare patterns of gene flow due to the absence of polymorphism in the parasite cytb marker used. Gomez-Diaz et al. (2011) detected large genetic variability in populations of Borrelia garinii across an entire oceanic region but found it to be unstructured despite the presence of host races within the tick vector across this region (McCoy et al. 2005b). In both studies, the parasite was a generalist, and both vectors, the tick and hippoboscid fly, may feed on several species of bird. Dispersal patterns of parasite and hosts are therefore not expected to compare one-to-one; dispersal opportunities provided by unsampled host species could cause unexplainable discrepancies in the patterns of gene flow.

The goal of this study is to test the hypothesis that heteroxenous parasite dispersal patterns mimic those of its most vagile host (Jarne \& Théron 2001) by comparing and quantifying the patterns of gene flow of a specialist parasite, its vector and its vertebrate host. To this end, we use a haemosporidian parasite, Polychromophilus melanipherus (Apicomplexa: Haemosporida), a malaria-like blood parasite of bats. In its European range, it mainly parasitizes Miniopterus schreibersii (Chiroptera: Miniopteridae), but is known to infect other members of the Miniopteridae in Africa (Garnham 1966; Lanza 1999; Duval et al. 2012). Nycteribia schmidlii (Diptera: Nycteribiidae) is a small wingless bat fly that is a host-specific ectoparasite of M. schreibersii (Theodor 1957), although can occasionally be found on other bat species sharing the same cave (Lanza 1999). Although it has never been formally demonstrated, it is the suspected vector of $P$. melanipherus, as it is the most common bat fly of $M$. schreibersii (Estrada-Peña \& SerraCobo 1991), occurring throughout its European range (Theodor 1967) and is closely related to Nycteribia kolenatii, the confirmed vector of Polychromophilus murinus (Gardner et al. 1987). Using microsatellites, we first tested which of the two host species is more vagile, the bat M. schreibersii or the bat fly N. schmidlii. We then compared both the dispersal patterns of host and vector with that of the parasite P. melanipherus using mitochondrial markers, identifying whether the dispersal patterns mimic more those of its vector or its host.

\section{Methods}

\section{Sampling}

Sampling was carried out in May 2011 (Croatia, Portugal, Spain, Switzerland) and August/September 2012 (Italy, Slovakia, France, Switzerland) on 23 different sites across southern and Central Europe (Table 1, Fig. 1A). Miniopterus schreibersii were captured at their roosts either upon emergence using mist nets and harp traps at dusk, or by entering the roosts at day time and collecting them while they were resting. Young of the year, born in June/July, were not sampled. Bat flies were looked for by blowing through the fur of the bat, collected using soft forceps and immediately stored on $96 \%$ ethanol. 
Table 1 Overview of sampling effort and infection rates

\begin{tabular}{|c|c|c|c|c|c|}
\hline & Name & Region & $N$ & $N$ inf. & $N$ nyct. \\
\hline Pt1 & Soïdos & Portugal & 11 & $10(10)$ & 5 \\
\hline Pt2 & Preguiça & Portugal & 11 & $11(11)$ & 8 \\
\hline $\mathrm{Pt} 3$ & Cercal & Portugal & 15 & $12(11)$ & 6 \\
\hline Pt4 & Nabão & Portugal & 15 & $15(11)$ & 22 \\
\hline Es1 & Llaberia & Spain & 15 & $15(12)$ & 24 \\
\hline Es2 & Collserola & Spain & 15 & $14(11)$ & 14 \\
\hline Es3 & Montnegre & Spain & 14 & $13(10)$ & 21 \\
\hline Es4 & Savassona & Spain & 15 & $11(8)$ & 9 \\
\hline Es5 & Les Gavarres & Spain & 14 & $14(10)$ & 7 \\
\hline Fr1 & St-Médard & France & 15 & $7(7)$ & 3 \\
\hline Ch1 & Baulmes & Switzerland & 16 & $9(8)$ & 0 \\
\hline It1 & Monte Catini & Italy & 15 & $6(4)$ & 43 \\
\hline It2 & Riolo Terme & Italy & 15 & $9(8)$ & 17 \\
\hline It3 & San Marino & Italy & 15 & $12(12)$ & 29 \\
\hline It 4 & Velo Veronese & Italy & 3 & $1(1)$ & 9 \\
\hline Si1 & Marzamemi & Sicily & 16 & $11(11)$ & 0 \\
\hline Hr1 & Karin Gornji & Croatia & 6 & $6(6)$ & 2 \\
\hline $\mathrm{Hr} 2$ & Kijevo & Croatia & 15 & $13(11)$ & 5 \\
\hline $\mathrm{Hr} 3$ & Drnis & Croatia & 15 & $12(11)$ & 9 \\
\hline $\mathrm{Hr} 4$ & Rumin & Croatia & 9 & $8(5)$ & 2 \\
\hline Sk1 & Chvalovská jaskyňa & Slovakia & 15 & $10(9)$ & 17 \\
\hline Sk2 & Nandraž Bradlo & Slovakia & 15 & $4(4)$ & 16 \\
\hline Sk3 & Drienovecká jaskyňa & Slovakia & 15 & $8(8)$ & 10 \\
\hline
\end{tabular}

$N$, number of Miniopterus schreibersii hosts sampled; $N$ inf., number of bats infected with the parasite Polychromophilus melanipherus (number of single clone infections); $N$ nyct., number of Nycteribia schmidlii bat fly vectors collected.

A wing biopsy was taken using a 1-mm gauge punch (Stiefel) and stored in $96 \%$ ethanol. Approximately $15 \mu \mathrm{L}$ of blood was taken by puncturing the uropatagial vein with a $0.5-\mathrm{mm}$ gauge needle (Neolus, Terumo). Blood beads forming on the patagium were captured by pipetting or using a heparinized glass microcapillary tubes (Marcel Blanc \& Cie) and ejected on white blotting paper (3MM, Whatman) which was left to dry and later stored in separate envelopes. Wounds were treated with haemostatic cotton until bleeding had stopped after which bats were released at the site of capture. All captures were carried out in accordance with local regulations.

\section{DNA extraction}

DNA was extracted from the blood-soaked blotting paper using the DNeasy Blood and Tissue kit (Qiagen) according to the manufacturer's tissue protocol with the following exceptions. A fragment of paper of $\sim 10 \mathrm{~mm}$ in diameter was cut in smaller pieces using sterile scissors, added to a 2-mL tube (Eppendorf) containing $360 \mu \mathrm{L}$ of ATL buffer and incubated at $90{ }^{\circ} \mathrm{C}$ for $15 \mathrm{~min}$. After cooling down, $40 \mu \mathrm{L}$ of proteinase $K$ was added to each sample and left for overnight digestion at $56{ }^{\circ} \mathrm{C}$. At the final step, $55 \mu \mathrm{L}$ elution buffer was added to the filter and samples were incubated at $37{ }^{\circ} \mathrm{C}$ for $15 \mathrm{~min}$. before centrifuging. This step was repeated once to maximize recuperation. For a subset of individuals, extra DNA was extracted from the wing biopsy following the same tissue protocol with the addition of an initial rinsing phase where ethanol was removed from the tissue by soaking the biopsy in $1 \mathrm{~mL}$ of pure MilliQ water (Millipore).

For all collected bat flies, species was determined following the key by Theodor (1967). All specimens identified as Nycteribia schmidlii were rinsed of their ethanol by soaking in $1 \mathrm{~mL}$ of pure MilliQ water (Millipore) at room temperature for $2-3 \mathrm{~h}$. Because females most often carry a developing larva, their abdomens were removed to prevent paternal contamination. Afterwards, individual flies were triturated using sterile pestles. After an overnight digestion, the standard Biosprint 96 tissue protocol (Qiagen) was followed.

\section{Marker amplification}

To determine the genetic structure of M. schreibersii in Europe, all individuals were genotyped at 14 polymorphic microsatellite loci (Table S1, Supporting information; Miller-Butterworth et al. 2002; Wood et al. 2011). Primers, PCR thermal profiles and reagent 

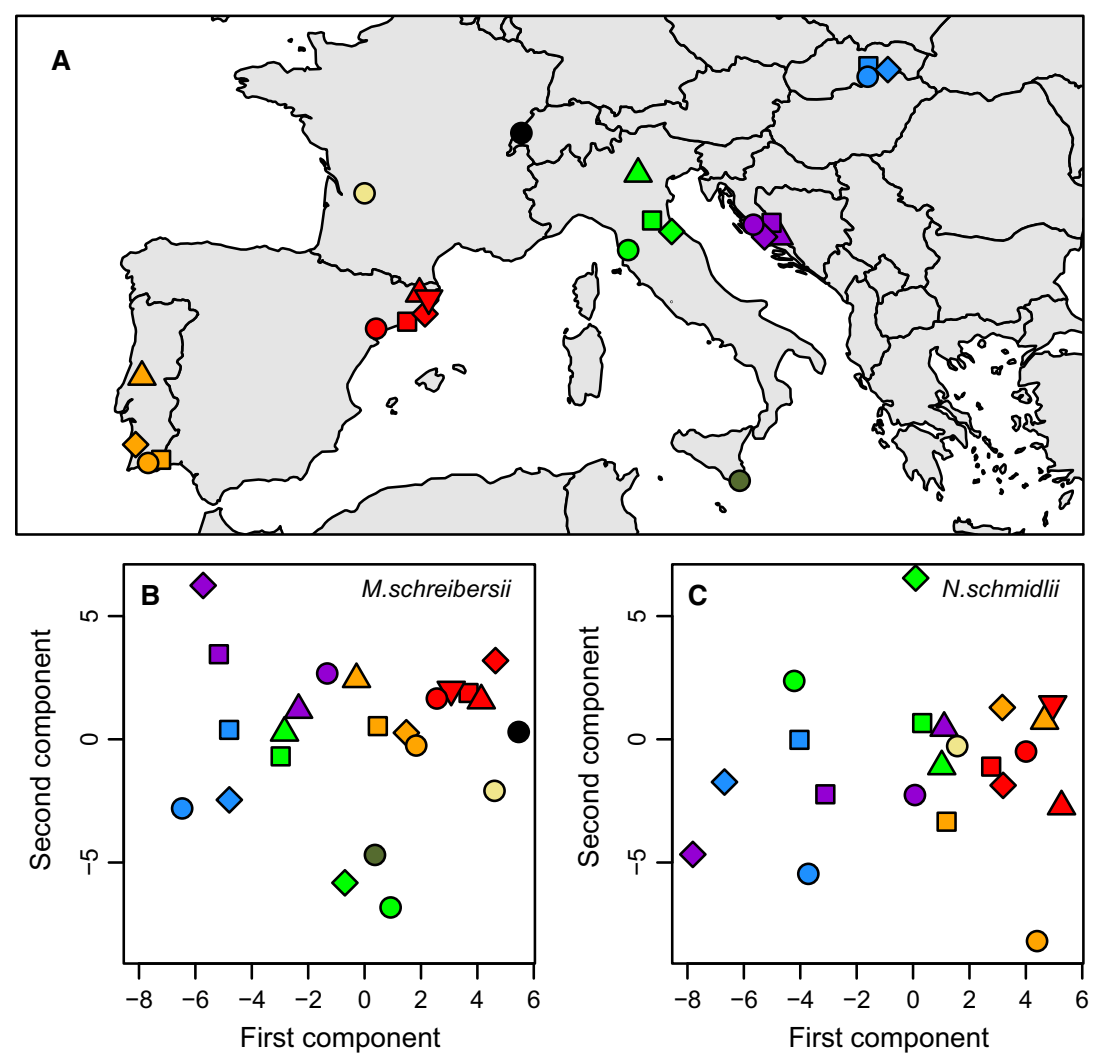

concentrations can be found in Tables S1-S3 (Supporting information). PCR products were controlled on an agarose gel 2\% under UV light. Four post-PCR multiplexes were realized after different tests by adjusting the ratio of each reaction in a final volume of $3 \mu \mathrm{L}$ (Table S1, Supporting information). PCR products were typed in an ABI Prism 3100 sequencer (Applied Biosystems).

For N. schmidlii, 10 new microsatellite markers were developed commercially (Ecogenics, Zurich-Schlieren, Switzerland). Size selected fragments from genomic DNA were enriched for microsatellite content using magnetic streptavidin beads and biotin-labelled CT and GT repeat oligonucleotides. The microsatellite-enriched library was analysed on a Roche 454 platform using the GS FLX titanium reagents. Reads were selected for tetra- or trinucleotide repeats of at least 6 units long or a dinucleotide repeat of at least 10 units long. Of these reads, 1173 were suitable for primer design, which resulted in primers for 10 new markers (Table S4, Supporting information).

The 10 new microsatellite markers for N. schmidlii were combined in two multiplexes, each reaction combining five loci each. See Tables S2 and S3 (Supporting information) for the thermal profiles and reagent concentrations, respectively. Post-PCR reaction products were diluted $6 \times$ of which $3 \mu \mathrm{L}$ was finally genotyped on an ABI prism 3100 sequencer.
Fig. 1 The geographic distribution of sampling sites and principle component analysis (PCA) results. (A) Map of southern Europe with sampling sites; $(B, C)$ Scatterplots of populations according to the first two axes of the PCA based on the microsatellite allele frequencies. (B) The host Miniopterus schreibersii; (C) The vector Nycteribia schmidlii. No vectors were collected in Sicily and Switzerland. PC1 was significant for both species, PC2 only for M. schreibersii. Symbols coloured by region and shaped according to population abbreviation (Table 1): 1 = circles, 2 = squares, 3 = diamonds, $4=$ upwardpointing triangles, $5=$ downward-pointing triangles.
The detection of Polychromophilus melanipherus infection in the vertebrate host's blood was carried out by amplifying a 705-bp cytochrome $b$ fragment (cyt $b$ ) of the parasite following a nested PCR protocol. Primers, reagents and PCR temperature profile were taken from Megali et al. (2011; Tables S2 and S3, Supporting information). Bands were run on a $1 \%$ agarose gel stained with ethidium bromide and were visualized under UV light. Each sample was tested in duplicate. If, after retesting, the sample continued to give ambiguous results, we considered it an infection of very low intensity and therefore included it in further analysis. Samples that, after retesting gave no bands at all, were presumably contaminated during the previous run, considered negative, and left out of further analyses. Positive samples were either purified with the Wizard PCR clean-up system (Promega) and sequenced locally on a ABI Prism 3100 genetic analyser (Applied Biosystems), or sent to a commercial agent for sequencing (Microsynth, Switzerland).

To compare the molecular variability of the parasite with that of the vector and host, also a mitochondrial gene fragment of vector and host was amplified. Based on preliminary tests with several candidate markers and 10 samples, we selected for each species a marker which produced a workable level of diversity. For N. schmidlii, 408 bp of the $16 \mathrm{~S}$ ribosomal subunit (16S) 
was amplified using the primers fw: 5'-CGC CTG TTT AAC AAA AAC AT-3' and rv: 5'-TGA ACT CAG ATC ATG TAA GAA A-3' (Petersen et al. 2007). For M. schreibersii, $310 \mathrm{bp}$ of the mitochondrial control region (CR, often referred to as 'd-loop') was amplified using the forward primer 5'-CAT CTG GTT CTT ACT TCA GG-3' (Fumagalli et al. 1996) and a newly designed reverse primer 5'-GTG CAC AGT CGT AAT CTC $-3^{\prime}$. Conditions and reagents for both amplifications can be found in Tables S2 and S3 (Supporting information). Purification and sequencing using the forward primers was performed by a commercial agent (Microsynth).

\section{Microsatellite analyses}

Microsatellite allele sizes were scored and sized by hand with the aid of the software GENEMAPPER (Applied Biosystems). MICRO-CHECKER v2.2.3 (Van Oosterhout et al. 2004) was used to investigate large allelic drop out, stuttering and null alleles. The software FSTAT v2.9.3.2 (Goudet 2001) was used to test for linkage disequilibrium between loci. Deviation from Hardy-Weinberg equilibrium across all markers and populations was tested for with the $\chi^{2}$-test from the 'GENETICS' package in R v3.0.1 (R Core Team 2012). Using the R-package HIERFSTAT v. 0.04-10 (Goudet 2005), the observed heterozygosity, within population and overall gene diversity, allelic richness and within population heterozygote deficiency $F_{\text {IS }}$ were calculated for each species.

For both the bats and the bat flies, a hierarchical population structure was assumed where sampling sites were clustered in regions (Table 1). To partition the components of genetic variation among regions and sites, a hierarchical analysis of variance (ANOVA) was performed using the HIERFSTAT function varcomp.glob. Significance of the variance components was tested using likelihood ratio G-statistic permutation tests from the same package. To make the fixation indices comparable between species, $F^{\prime}$-values, corrected for maximum polymorphism, were calculated by dividing each F-value by its theoretical maximum (Jost 2008).

A pattern of isolation by distance between sampling sites was examined by looking at the relation between the pairwise $F_{\mathrm{ST}}$ 's, $x /(1-x)$ transformed and the natural logarithm of the overland geographical distances (Rousset 1997). The correlations between the genetic and geographical distance matrices were tested using a mantel test (1000 permutations) using the mantel function from the R-package ADE4 v1.5-2 (Dray \& Dufour 2007).

For both the bats and bat flies, a principle component analysis (PCA) was performed on the population allele frequencies, weighted by population size to prevent small populations from having disproportional effects. The eigenvectors were extracted using the function dudi.pca from the ADE4 package. Significance of the inertia of the first two components was calculated by permuting the population assignment of each individual and recalculating the inertia 1000 times using a custom script in $\mathrm{R}$.

The most likely number of clusters in each species' European population was estimated within the Bayesian framework of STRUCTURE v2.3.4 (Pritchard et al. 2000). A model with admixture and correlated alleles (Falush et al. 2003) was used to assign individuals to any of the predestined number of clusters, ranging from 1 to 8 , based on the number of regions sampled. The sample location was not used as a prior. Each simulation had a burn-in of 50000 generations and data collection for 100000 generations, and 10 replicates were performed for each parameter setting. The most likely number of clusters was determined by looking at the raw log likelihood, as well as $\Delta K$, the ratio of the absolute rate of change of the log likelihood and its standard deviation (Evanno et al. 2005), as implemented in Structure Harvester (Earl \& Vonholdt 2012).

\section{Mitochondrial sequence analysis}

The chromatographs of all mtDNA sequences were manually checked, edited and aligned with the software MEGA 5.03 (Tamura et al. 2011). Identification and characterization of haplotypes and the sequence diversity of the sampling sites were carried out with the aid of the PEGAS v0.4-5 package in $\mathrm{R}$ (Paradis 2010). A minimumspanning network was created of the haplotypes for each of the three species in TCS v1.21 (Clement et al. 2000). Neutral evolution of each fragment was assessed by calculating Tajima's $D$ and tested for significant deviation from zero using the function tajima.test from PEGAS, customized to take into account insertion-deletions (indels).

As with the microsatellite analyses, the components of genetic variation were partitioned hierarchically, although not only based on the local haplotype frequencies, but also on their molecular distances, in an analysis of molecular variance (AMOVA; Excoffier et al. 1992). To calculate the molecular distance between sequences two different models of sequence evolution were used as follows: (i) the Kimura 2 parameter model of nucleotide evolution, ignoring indels (Kimura 1980) or (ii) the absolute number pairwise differences, where the presence of different sized indels, were considered a single difference, irrespective of their size difference in base pairs. Besides the AMOVA, a standard ANOVA (disregarding molecular distances among haplotypes) was performed allowing the identification of the effects of the 
evolutionary model, and again, the corrected $F^{\prime}$-values were calculated as well. The components of variation of each distance model were tested using the AMOvA randomization test from the ADE4 package.

ARLEQUIN v3.1 (Excoffier et al. 2005) was used to produce pairwise $\Phi_{\mathrm{ST}}$ distance matrices for each species. These population distance matrices were based on both the frequency and sequence data of the mitochondrial fragments and were used as measures of genetic distance between each sampling site for each species. These were used to test for isolation by distance between sites after transformation as described above for the microsatellites. Moreover, the bat and bat fly matrices were compared with their microsatellite counterpart using the mantel test (1000 permutations) from the ADE4 package. Lastly, the $\Phi_{\mathrm{ST}}$ distance matrix of P. melanipherus was compared with both nuclear and mitochondrial distance matrices of both M. schreibersii and N. schmidlii. To test for different dispersal patterns based on infection, the mantel test was repeated for M. schreibersii, including only individuals of the host population infected with $P$. melanipherus.

\section{Results}

\section{Miniopterus schreibersii and Nycteribia schmidlii: comparison of microsatellites}

Three microsatellite loci of M. schreibersii, MM01, MM29 and MM34, had only a single allele and were removed from any further analyses, leaving the total number of markers at 11. No signs of stuttering, allelic drop out or null alleles were found in the bat microsatellite data set and no loci showed signs of linkage. Eight populationlocus combinations were not in Hardy-Weinberg equilibrium after Bonferroni correction. However, these

Table 2 Hierarchical analysis of the variance components of genetic diversity of the host Miniopterus schreibersii and the vector Nycteribia schmidlii. Sampling site was the lowest hierarchical level, the middle level consisted of the regions, loosely following country borders (see Table 1)

\begin{tabular}{|c|c|c|c|c|}
\hline & $F$-value & CI & $P$ & $F^{\prime}$ \\
\hline \multicolumn{5}{|c|}{ The host M. schreibersii } \\
\hline$F_{\mathrm{ST}}$ & 0.0629 & $0.0482-0.0798$ & 0.001 & 0.1391 \\
\hline$F_{\mathrm{SC}}$ & 0.0203 & $0.0111-0.0311$ & 0.001 & 0.0449 \\
\hline$F_{\mathrm{CT}}$ & 0.0434 & $0.0332-0.0534$ & 0.001 & 0.0986 \\
\hline \multicolumn{5}{|c|}{ The vector N. schmidlii } \\
\hline$F_{\mathrm{ST}}$ & 0.0053 & $0.0012-0.0108$ & 0.014 & 0.0240 \\
\hline$F_{\mathrm{SC}}$ & -0.0010 & $-0.0040-0.0015$ & 0.497 & -0.0046 \\
\hline$F_{\mathrm{CT}}$ & 0.0063 & $0.0019-0.0137$ & 0.002 & 0.0284 \\
\hline
\end{tabular}

CI, 95\% confidence interval of the $F$-value; $P$, associated $P$-value based on 1000 permutations. were scattered randomly across the total 253 comparisons and were therefore left in the data set.

In the N. schmidlii data, all markers were polymorphic. However, two loci, Ns134 and Ns719, produced null alleles consistently across populations. These loci were removed from any further analyses. No marker pair showed linkage and all markers were in HardyWeinberg equilibrium for each population.

The bat fly markers tended to be more diverse than those of the bats. Both the absolute number of alleles and gene diversities $\left(H_{\mathrm{S}}, H_{\mathrm{T}}\right)$ seemed higher in the flies (Table S5, Supporting information). Bat flies showed slightly higher values of $F_{\mathrm{IS}}$, both across markers and populations (Tables S5 and S6, Supporting information). At higher hierarchical levels, $F_{\mathrm{CT}}$ and $F_{\mathrm{ST}}$ were 8-10 times higher in bats than bat flies (Table 2). Corrected for the lower gene diversity, the bats still show a sixfold higher level of isolation (Table 2: $F^{\prime}$-values). Within region, the differentiation between sampling sites was only significantly different from zero in the bats, not in the bat flies. In both species, the differentiation among sites followed an isolation-by-distance pattern (mantel test; M. schreibersii: $r=0.396, \quad P=0.001 ; \quad N$. schmidlii: $r=0.275, P=0.002$; Fig. S1A,B, Supporting information) but had twice the explanatory power in the bat $\left(r^{2}=0.157\right)$ than in the bat fly $\left(r^{2}=0.076\right)$. In contrast, the population isolation patterns of each species did not correlate with each other (mantel test; $M$. schreibersii vs. N. schmidlii: $r=0.020, P=0.434$; Fig. S1C, Supporting information).

For both M. schreibersii and N. schmidlii, the first principle component (PC1) explained a significant amount of the allelic variation observed in the European populations (permutation tests: $M$. schreibersii: inertia $=0.148$; $P=0.001 ; \quad$ N. schmidlii: inertia $=0.114 ; \quad P=0.006$ ). In both species, this axis demonstrated an east-west pattern, except Portugal remarkably taking up a somewhat central position (Fig. 1B,C). Inertia of PC2 was significant for M. schreibersii, but not N. schmidlii (permutation tests: $M$. schreibersii: inertia $=0.105, P=0.001 ; N$. schmidlii: inertia $=0.081 ; P=0.884)$. PC2 separates the more western and southern Italian sites (together with the Sicilian site), from the other M. schreibersii samples (Fig. 1B). Overall, the M. schreibersii samples were more clumped by region whereas the $N$. schmidlii sites were more mixed among regions.

Following the method of Evanno et al. (2005), $K=2$ was the most likely number of clusters in the European population of $M$. schreibersii $\left(\Delta K_{2}=31.01\right.$, mean $\ln L=-8210.01$, Fig. S2, Supporting information). According to the structure plot, the Spanish, French and Swiss sites formed one cluster and all sites more east a second. The Portuguese samples were identified as an admixture of the two clusters. In contrast with the 
bat host, the most likely number of clusters for the $N$. schmidlii data was $K=1$. Simulations with a single cluster produced the highest absolute log-probability (mean $\operatorname{lnL}=-9068.26$ ), which decreased monotonically with an increasing number of suggested clusters.

\section{Host, vector, parasite: mtDNA sequence description}

Of the 310 bats examined, 231 proved PCR-positive for Polychromophilus melanipherus, resulting in a cross-European infection rate of $74.5 \%$ (Table 1, Fig. S3, Supporting information). After sequencing, 32 of the 231 infections (14\%) had multiple ambiguous base calls, indicating these hosts were infected with multiple strains of P. melanipherus at the time of sampling. Because haplotypes could not be called unambiguously in these cases, the multiple infections were disregarded in the consecutive analyses.

Sequence analysis of the $P$. melanipherus cyt $b$ fragment revealed eight haplotypes in the European population, based on 22 segregating sites. The 310-bp CR fragment of M. schreibersii had 51 segregating sites and 103 haplotypes. The 455-bp $16 \mathrm{~S}$ fragment of N. schmidlii had 32 haplotypes, based on 15 segregating sites plus 3 indels: a single adenine indel, an adenine-thymine indel and an indel consisting of $0-5$ repeated thymines. Independent reamplification and resequencing confirmed these indels. For both M. schreibersii (51 of 103) and $N$. schmidlii (15 of 32) approximately half of the haplotypes were singletons, found in only 1 individual. All unique haplotype sequences of $P$. melanipherus (Accession nos KJ131270-KJ131277), M. schreibersii (Accession nos KJ131278-KJ131380) and N. schmidlii (Accession nos KJ131381-KJ131412) were deposited at the publicly accessible online database GenBank (http://www.ncbi.nlm. nih.gov/genbank/).

Both haplotypic diversity and nucleotidic diversity were of the same order of magnitude for each species (Table S7, Supporting information). Despite having the shortest fragment, M. schreibersii had haplotypic diversity close to unity, whereas the vector and parasite had very similar, slightly lower values. The reverse trend had been observed in the microsatellites, where heterozygosity levels were higher in the vector than in the host. The vector $N$. schmidlii's gene fragment had the lowest nucleotide diversity of the three species. However, this is partly an artefact caused by the indels in the sequence, which are ignored when calculating nucleotide diversity.

The minimum-spanning network of P. melanipherus consisted of the eight haplotypes loosely connected by branches varying in length from 1 to 12 mutational steps with no alternative paths between haplotypes (Fig. 2D). The most numerous haplotypes were also common across all regions, whereas a few rare haplotypes were specific to certain locations. Both the bat and bat fly minimum-spanning networks formed large strongly interconnected nets. Most haplotypes were separated by only 1-3 mutational steps, and many alternative paths existed between haplotypes. Because of the

A

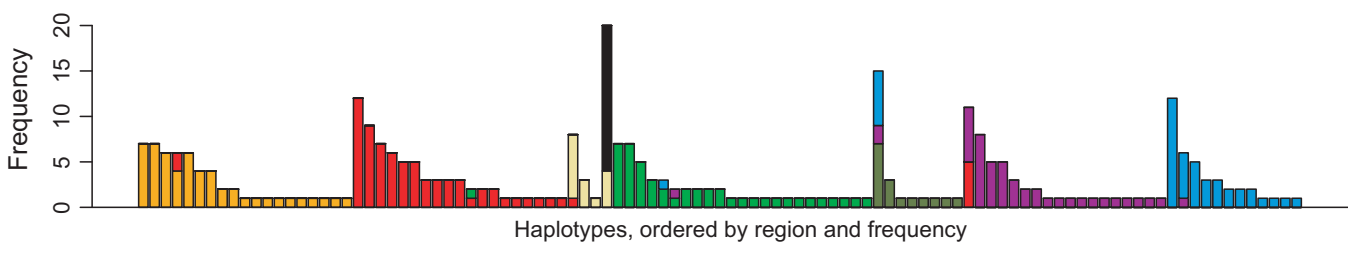

D
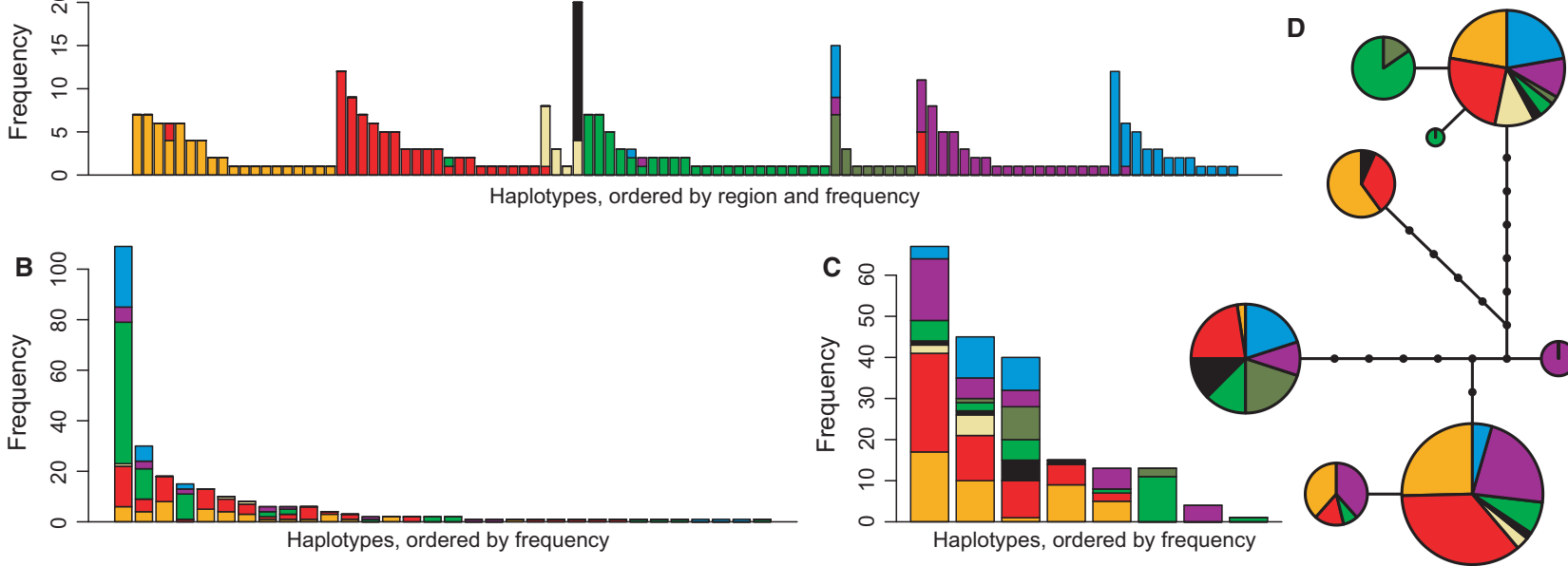

Fig. 2 Frequency of each mitochondrial haplotype and distribution among the different regions sampled. Colour coding according to region (see also Fig. 1). (A) 103 haplotypes of the host Miniopterus schreibersii; (B) 32 haplotypes of the vector Nycteribia schmidlii; (C) eight haplotypes of the parasite Polchromophilus melanipherus. (D) A minimum-spanning network of the eight cytb haplotypes of P. melanipherus in Europe. Haplotypes are represented by pie charts, the colour represents the regions where this haplotype was found and the size of the pie chart is relative to the total frequency of the haplotype. Each line represents a single nucleotide mutation, and black dots represent unobserved hypothetical haplotypes. 
many haplotypes and many connections, no clear minimum-spanning network figures could be produced; however, Fig. 2A-C show the frequency and distribution of haplotypes across the different regions. None of the bats' CR haplotypes were shared among all sites; most of them were unique to a specific region. In contrast, the bat flies' 16S fragment had one extremely prevalent haplotype, followed by a few considerably less prevalent haplotypes, but all except the rarest were shared among regions (Fig. 2B).

Selection might be in part responsible for the marked differences in minimum-spanning networks. Indeed, the mtDNA fragment of $M$. schreibersii and N. schmidlii showed no signs of selection (M. schreibersii: $D=-1.115, \quad P=0.265 ; \quad N$. schmidlii: $\quad D=-0.370$, $P=0.712$ ). In contrast, the cytb fragment of $P$. melanipherus had a high segregating-sites-to-haplotypes ratio $(D=2.178, P=0.029)$, a sign of balancing selection or population contraction.

When not correcting for mutations, the host M. schreibersii showed the highest levels of population differentiation ( $F^{\prime}$-values, Table 3: 'Equidistant'), compared to vector and parasite. When mutations are considered, the contrast between the species' population differentiation values becomes less pronounced (Table 3: 'Pairwise differences', 'Kimura 2p'). The vector $N$. schmidlii had the lowest (and nonsignificant) withinregion differentiation $\left(\Phi_{\mathrm{SC}}\right)$, but slightly higher levels of isolation among sites compared to parasite and host $\left(\Phi_{\mathrm{ST}}, \Phi_{\mathrm{CT}}\right)$. The host $M$. schreibersii has the highest, significant, levels of within-region isolation $\left(\Phi_{\mathrm{SC}}\right)$, whereas the parasite $P$. melanipherus is overall the least differentiated among sites. The two modes of nucleotide evolution differed very little in their effect on the $\Phi$-statistics of parasite and host (Table 3). Notable exception was seen with the vector, where a clear difference is shown between the Kimura model (ignoring indels) and pairwise differences (including indels in the distance matrix).

The pairwise $\Phi_{\mathrm{ST}}$ matrices of N. schmidlii and M. schreibersii were produced using pairwise number of differences as the mutation model. The pairwise genetic distance matrix of $P$. melanipherus was calculated using no mutation model, as the differences in $F$-values between the mutation models were minimal (Table 3 ). The patterns of population differentiation based on the mtDNA fragment agreed with the patterns based on microsatellites in both carrier species (mantel test, microsatellite $F_{\mathrm{ST}}-$ mtDNA $\Phi_{\mathrm{ST}} ; M$. schreibersii: $r=0.333$, $P=0.004 ;$ N schmidlii: $r=0.294, P=0.003)$. As with the microsatellites, the mitochondrial fragments of both the bat (mantel test; $r=0.182, P=0.005$ ) and the bat fly (mantel test; $r=0.475, P=0.001$ ) demonstrated an isolation-by-distance pattern. Visual inspection further indicates that this pattern is mainly present on the long, interregional distances; the left of the graph, representing sites separated by short distances, suggests no relation between the genetic and geographic distances within region (Fig. S4A,B, Supporting information). The malaria parasite, however, showed no such geographical pattern (mantel test; $r=0.072, P=0.191$; Fig. S4C, Supporting information).

\section{Comparisons between parasite and vector or host genetics}

The parasite's genetic distance matrix did not correlate with either that of the host or the vector; neither when

Table 3 Analysis of molecular variance of the mtDNA fragments, for three mutation models

\begin{tabular}{|c|c|c|c|c|c|c|c|c|c|c|}
\hline & \multicolumn{4}{|c|}{ Equidistant } & \multicolumn{3}{|c|}{ Pairwise differences } & \multicolumn{3}{|c|}{ Kimura $2 p$} \\
\hline & $F$-value & $\%$ & $P$-value & $F^{\prime}$ & $\Phi$-value & $\%$ & $P$-value & $\Phi$-value & $\%$ & $P$-value \\
\hline \multicolumn{11}{|c|}{ The host Miniopterus schreibersii } \\
\hline$F_{\mathrm{ST}}$ & 0.1914 & 80.86 & 0.0001 & 1 & 0.3245 & 67.55 & 0.0001 & 0.3231 & 67.69 & 0.0001 \\
\hline$F_{\mathrm{SC}}$ & 0.0961 & 8.6 & 0.0001 & 0.5067 & 0.1089 & 8.26 & 0.0001 & 0.1084 & 8.23 & 0.0001 \\
\hline$F_{\mathrm{CT}}$ & 0.1054 & 10.54 & 0.0001 & 1 & 0.2419 & 24.19 & 0.0001 & 0.2407 & 24.07 & 0.0001 \\
\hline \multicolumn{11}{|c|}{ The vector Nycteribia schmidlii } \\
\hline$F_{\mathrm{ST}}$ & 0.0885 & 91.15 & 0.0001 & 0.3318 & 0.4068 & 59.32 & 0.0001 & 0.502 & 49.8 & 0.0001 \\
\hline$F_{\mathrm{SC}}$ & -0.0007 & -0.07 & 0.6679 & -0.0028 & 0.0265 & 1.61 & 0.2178 & 0.0338 & 1.74 & 0.2283 \\
\hline$F_{\mathrm{CT}}$ & 0.0891 & 8.91 & 0.0007 & 0.3336 & 0.3906 & 39.06 & 0.0002 & 0.4846 & 48.46 & 0.0001 \\
\hline \multicolumn{11}{|c|}{ The parasite Polychromophilus melanipherus } \\
\hline$F_{\mathrm{ST}}$ & 0.163 & 83.7 & 0.0001 & 0.4948 & 0.1592 & 84.08 & 0.0001 & 0.1592 & 84.08 & 0.0002 \\
\hline$F_{\mathrm{SC}}$ & 0.0531 & 4.69 & 0.0475 & 0.1456 & 0.0527 & 4.68 & 0.0755 & 0.0526 & 4.67 & 0.0752 \\
\hline$F_{\mathrm{CT}}$ & 0.1161 & 11.61 & 0.0001 & 0.4087 & 0.1125 & 11.25 & 0.0019 & 0.1125 & 11.25 & 0.0027 \\
\hline
\end{tabular}

Significant values are in bold. 
the distances were based on microsatellites, nor on mtDNA (Table 4, Fig. 3, Tables S8-S10, Supporting information). This was also true when only actual 'hosts' were used, that is, when only the bat host genotypes were included in the distance table that were actually infected with the parasite (Table 4). In contrast to their microsatellites, the host and vector mtDNA distance matrices did correlate significantly with each other (mantel test; $r=0.529, P=0.001, r^{2}=0.280$ ), although this correlation disappeared when corrected for geographic distance (mantel test; $r=0.077$, $P=0.164)$.

\section{Discussion}

Parasite gene flow is dependent on both vector and host dispersal behaviour. In this study, we determined the population genetic structure of the haemosporidian parasite Polychromophilus melanipherus and tested the hypothesis that its structure matches that of its most vagile host species. We demonstrated that it is the wingless vector Nycteribia schmidlii that is most vagile and not the vertebrate host $M$. schreibersii. However, the pattern of genetic differentiation of P. melanipherus resembled neither that of the vector nor that of the host.

\section{Which is the most vagile species?}

When comparing the microsatellite data of the host M. schreibersii and the vector N. schmidlii, a clear pattern emerges. Although the $F_{\mathrm{ST}}$ was significant in both species, the detected levels of isolation were 10 times higher in the bat than in the bat fly, whose level of subdivision was close to zero. Even after correcting for the slightly higher levels of gene diversity in the bat fly, differentiation among bat populations remained higher. In contrast, at the mitochondrial marker, the regional

Table 4 Mantel test results correlating the parasite's Polychromophilus melanipherus pairwise genetic distances with those of its host Miniopterus schreibersii and its vector Nycteribia schmidlii

\begin{tabular}{lrc}
\hline Compared dataset & \multicolumn{1}{l}{$P$} & $P$-value* \\
\hline M. schreibersii & & \\
$\quad$ Microsatellites & 0.0427 & 0.364 \\
mtDNA & -0.0604 & 0.600 \\
Only infected bats & & \\
$\quad$ Microsatellites & 0.0959 & 0.215 \\
mtDNA & 0.0462 & 0.431 \\
N. schmidlii & & \\
Microsatellites & -0.0950 & 0.665 \\
mtDNA & 0.0740 & 0.182 \\
\hline
\end{tabular}

*Based on 1000 permutations. structuring was relatively similar for both species, with the exception that only the bat flies showed no significant structuring within regions. The clustering analysis and PCA confirm this pattern of stronger regional clustering in the bat hosts. Lastly, both species showed a pattern of isolation by distance, but this relation was much stronger in the bat. All these findings indicate that more genetic exchange takes place between populations of bat flies and less between the populations of their bat hosts. The N. schmidlii bat flies should therefore be considered the more vagile species in Europe.

However, the lower level of differentiation observed between bat fly sites might also be due to a possibly larger population size, reducing drift. Indeed most M. schreibersii would be carrying multiple N. schmidlii in summer making the bat fly population size correspondingly larger compared to the bat. Yet when hosts were sampled in spring (Croatia) and autumn (Switzerland) lower numbers of bat flies were found, averaging less than one fly per host (Table 1). Although bat fly sampling was not exhaustive in this study, and geography might be a confounding factor, seasonal bottlenecks have been observed in N. schmidlii (Estrada-Peña \& Serra-Cobo 1991) as well as other temperate Nycteribia species (Witsenburg et al. 2014). These severe bottlenecks should decrease the effective population size $N_{\mathrm{e}}$ and thereby increase relative levels of differentiation between bat fly populations. The shorter life cycle of temperate bat fly species (estimated at two generations per year; Reckardt \& Kerth 2006) should also increase relative population differentiation compared to the more slowly reproducing bat host. Despite these two possible factors, N. schmidlii had a lower fixation values, strongly suggesting more migration between vector populations compared to the host.

Despite their extensive seasonal migrations between roosts, actual dispersal events of marked M. schreibersii to a different population have very rarely been observed (Rodrigues et al. 2010). Both males and females are philopatric, in this case meaning faithful to their natal group, rather than to any geographic location, although females will always return to the same maternity roost (Rodrigues \& Palmeirim 2008; Rodrigues et al. 2010). Despite the philopatry of both sexes, the $\Phi_{\mathrm{ST}}$ of the maternally inherited CR fragment was much higher than the expected fourfold increase compared to the nuclear data. This implies that females disperse less than males (Ramos Pereira et al. 2009), which would contradict the behavioural studies. However, although males do not disperse to other groups, the seasonally migrating $M$. schreibersii do meet other local populations and mating at these meetings have been observed (Rodrigues et al. 2010), allowing for the transfer of nuclear, but not mitochondrial, genes to the other 

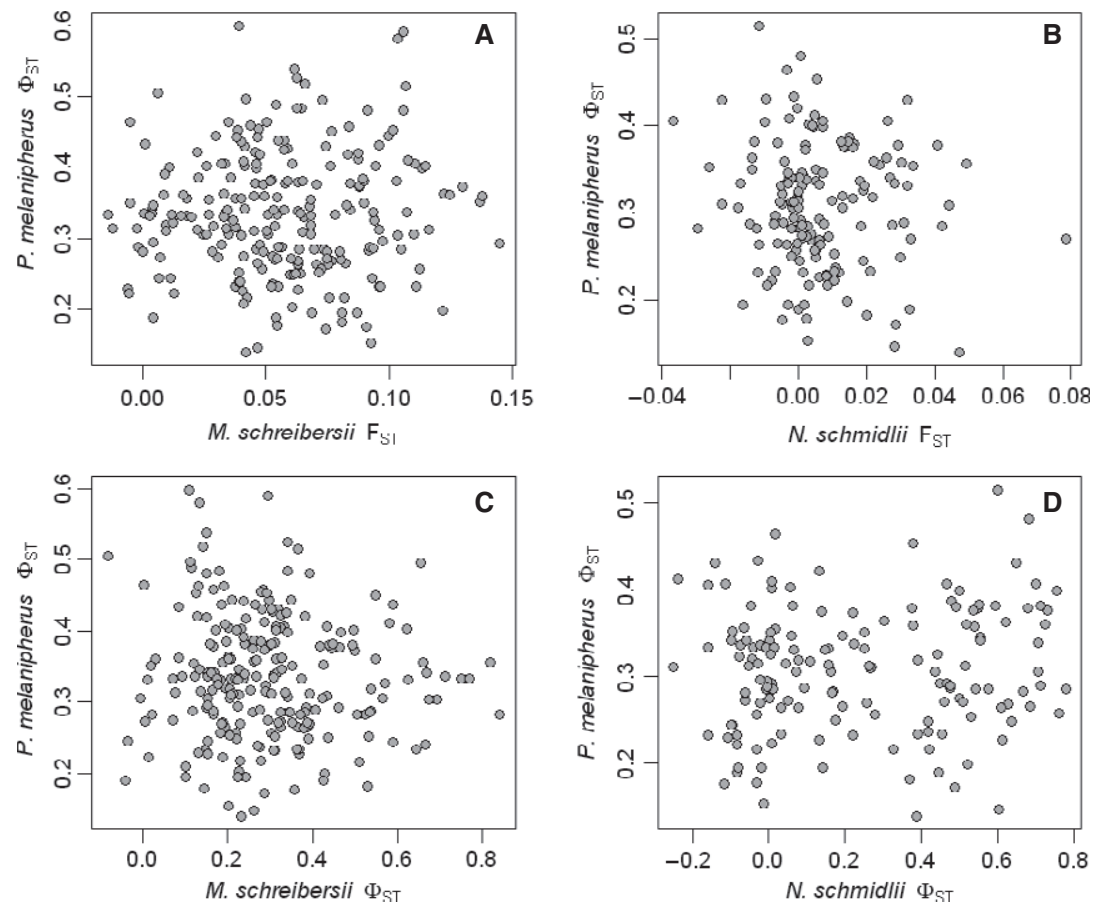

Fig. 3 Population differentiation patterns do not correlate between either host species or the malaria parasite. Microsatellite $\left(F_{\mathrm{ST}}\right.$, top row $\left.\mathrm{A}, \mathrm{B}\right)$ and mitochondrial sequence $\left(\Phi_{\mathrm{ST}}\right.$, bottom row $\left.\mathrm{C}, \mathrm{D}\right)$-based pairwise population genetic distances for the host Miniopterus schreibersii (left column A, C) and the vector Nycteribia schmidlii (right column B, D) compared with the pairwise population genetic distances for the parasite Polychromophilus melanipherus. See Tables S8-S10 (Supporting information) for the raw pairwise distance tables. population. Moreover, as the mitochondrial control region is the fastest evolving sequence of the mammalian genome (Fumagalli et al. 1996), the mutation model we used might not have been able to correctly capture all of the observed variation, artificially inflating the mtDNA fixation indices.

The exchange of bat flies appears much stronger than that of the bats' own genes. Although peak reproduction of bat flies is synchronized to that of the bat, N. schmidlii can reproduce throughout the season (Lourenço \& Palmeirim 2008). Moreover, hibernacula, shared among multiple subpopulations of $M$. schreibersii (Rodrigues \& Palmeirim 2008), could facilitate bat fly dispersal to other groups. But contact of bat subpopulations needs not be direct for N. schmidlii dispersal to occur. Roosting in the same cave at different time points would already allow for the exchange of bat flies, which leave their pupae to develop on the cave walls.

The N. schmidlii microsatellites and mitochondrial sequences agree that within regions all sites are strongly connected, with regular exchanges. But the between-region mitochondrial levels of fixation are 80 times the nuclear values. Again, our mutation model might not have effectively captured the mutational processes going on at the ribosomal RNA fragment. Yet, this large discrepancy also suggests a strongly reduced $N_{\mathrm{e}}$, caused by processes such as skewed sex ratios in the bat flies or strong female fly philopatry. Skewed sex ratios in favour of males are known to occur in bat flies (Dick \& Patterson 2008), but in this study, more female flies were found (but no exhaustive sampling was performed). Female bat flies have to leave their host frequently to deposit a pupa on the roost wall (Marshall 1970). This risky behaviour could lower the survival of females. On the other hand, this behaviour should cause the females to change hosts more often, increasing their dispersal likelihood. Sex differences in survival have not been observed in bat flies (Marshall 1970; Witsenburg et al. 2014). However, the performed experiments concerned short term off-host survival, yet these flies can live up to 6 months at least (Ryberg 1947 cited in Marshall 1970); sex differences in long-term survival might therefore very well be possible and have been observed with regard to haemosporidian infections in hippoboscid flies (Waite et al. 2012). Females might also be less likely to survive the winter season, causing bottlenecks in the mitochondrial genome. Bat fly female philopatry could also have caused the high level of differentiation of the mitochondrial gene, but to our knowledge, no study has looked into bat fly dispersal behaviour.

\section{Population structure of Polychromophilus melanipherus}

The cyt $b$ fragment of $P$. melanipherus was the only fragment that showed signs of past selection. It was also 
the only protein-coding gene in this study, which might explain the relatively few haplotypes separated by relatively long branches. The balancing selection indicates negative frequency-dependent selection, suggesting a co-evolutionary alternation of defences between the parasite and either the vector or host. However, how the mitochondrial cytochrome $b$, involved in the transmembrane transport of electrons and ultimately in ATP production, could be a target for host immune responses is not clear.

The four major haplotypes were shared among the different regions, with the other four being more geographically restricted. The bat fly also had its more frequent haplotypes shared among regions yet still had an isolation-by-distance pattern. The malaria parasite showed no such geographical pattern. Despite a relatively low expected $N_{\mathrm{e}}$ (infection rates $<1$, few multiclone infections, high expected inbreeding rate), and therefore high expected levels of differentiation among the parasite populations, the relative contribution of $\Phi_{\mathrm{CT}}$ to the parasites' structuring was low compared with those of vector and host. This suggests that the cross-region exchange of parasites is more frequent than either that of vector and host.

In avian malaria research, all cytb haplotypes are considered distinct nonrecombining genetic 'lineages', as they often have nonoverlapping host distributions (Bensch et al. 2000) and show linkage with nuclear haplotypes (Bensch et al. 2004). Although host distributions clearly overlapped in the case of P. melanipherus, we have no data on linkage with nuclear haplotypes. If our haplotypes actually represented cryptic species, gene diversity for each species should actually be higher, resulting in lower fixation values and therefore even more panmictic parasites. However, if a species were defined by its genetic haplotype, it will always be panmictic, as it would be genetically identical across its range by definition. The host distribution of the P. melanipherus haplotypes suggests they are a single species, but nuclear markers are required to determine whether they are truly recombining.

\section{Does the parasite mimic the vector's dispersal patterns?}

As stated by Wright (1951), $F_{\mathrm{ST}}$ is inversely related to the estimated number of migrants $N_{\mathrm{m}}$. With its lower $F_{\mathrm{ST}}, N$. schmidlii provides a higher number of migrants between demes compared to M. schreibersii. Even if a majority of these migrants was male, as suggested by the mtDNA, both male and female Nycteribiidae take bloodmeals and can therefore transmit P. melanipherus. The bat fly N. schmidlii should therefore be the main agent transporting the parasite between demes, but only if infection rates among bats and bat flies are equal. Although we did not measure the infection status of $N$. schmidlii here, the infection rate of its sister species Nycteribia kolenatii with the Polychromophilus murinus parasite was $17 \%$, four times lower than of its host M. daubentonii (Witsenburg et al. 2014). If similar rates were to apply to this system, the effective number of parasite dispersal events caused by vector or host might approach each other. However, the parasite's population structure neither resembled that of the bat fly vector, nor that of the bat host. And because the dispersal patterns of host and vector were correlated at the mitochondrial level, it is unlikely that the parasite's pattern would lie somewhere 'in the middle' of its two hosts, as an average of their patterns of gene flow.

An alternative hypothesis to the idea that a parasite's population structure should resemble that of its most vagile host is a simple additive model where the parasite's gene flow is represented by the added effects of host and vector dispersal patterns. Indeed, there is no a priori argument for ignoring any effect which an intermediate host might have on the parasite's dispersal. If one of the two hosts is a stationary, highly structured species, this hypothesis should produce the same results as Jarne and Théron's hypothesis of mimicking the most vagile species. However, if both host species show a certain degree of genetic exchange, the results of the two models should radically differ. Whereas the original hypothesis would expect the parasite's geneflow pattern still to be similar to that of the most vagile host, our proposed alternative states the parasite's gene flow should be higher than that of either host.

The fixation indices of P. melanipherus were lower than that of either host, but due to different expected mtDNA mutation rates, we should be cautious of direct comparisons. However, the absence of an isolation-bydistance pattern in the blood parasite, in contrast with either host, indicates that P. melanipherus does have higher levels of gene flow than either host. This lack of differentiation between parasite populations due to higher gene flow could also have caused the apparent absence of correlation between parasite and host populations.

A possible cause for the lack of correlation between the population structures of the parasite and its host and vector might also be the presence of a second vector. Penicilidia conspicua is a large bat fly species that can also be found on M. schreibersii (Theodor 1967). However, we found this species at lower intensities or not at all, and it was therefore considered a less likely candidate vector. Moreover, the dissection of 26 individuals revealed no oocysts. If $P$. conspicua would also transmit P. melanipherus, we would expect an overall 
increase in gene flow for the parasite relative to the vector, which might be the case in our study.

Dispersal rate heterogeneity within species, where infected individuals of a species show different dispersal behaviours compared to their uninfected counterparts, could have caused the discrepancy between host and parasite gene flow. However, N. kolenatii bat flies carrying $P$. murinus have lower survival than their uninfected counterparts (Witsenburg et al. 2014), which should reduce dispersal of infected flies, increasing the parasite population differentiation. No clear effects of a Polychromophilus infection on its bat host have been found as of yet, but an increase in migration behaviour of infected bats, for example being expelled from the current colony as a form of infection avoidance, could cause the parasite to be a more effective disperser than its host population (Curtis 2014).

We cannot say how much the discrepancy between hosts and parasite genetic structure is caused by the specific gene histories. As seen with N. schmidlii, the message from different types of markers can be markedly different. A comparison between parasite and hosts with microsatellites should give a higher resolution picture of geneflow patterns, but despite multiple efforts, we did not manage to develop microsatellite markers for P. melanipherus. Only once have microsatellites been used to compare dispersal patterns of a parasite with its two hosts (Prugnolle et al. 2005). Yet, as in our study, the authors found no correlation between their parasite (the trematode Schistosoma mansoni) and its little dispersing intermediate mollusc host Biomphalaria glabrata, nor with its definite host the rat Rattus rattus. Although undeniably dispersed by the rat, no apparent correlation existed between parasite and host gene flow. Only the genetic distance between the trematodes' infrapopulations showed a positive relationship with the shared allelic distance of each rat. Instead of a shared dispersal patterns, this might rather suggest a gene-by-gene model of co-evolution between parasite and host (Prugnolle et al. 2005).

To our knowledge, the Prugnolle et al. (2005) study is the only other study where a quantitative comparison has been made between parasite and both hosts. Blasco-Costa \& Poulin (2013) found in a meta-analysis of gene flow of trematode species that the most important factor influencing parasite gene flow was final host's mode of dispersal (aquatic or terrestrial/by air), although no traits of intermediate hosts were tested. A more quantitative comparison was made between the malaria parasite Plasmodium falciparum and its mosquito host Anopheles gambiae in Kenya. In concordance with the additive effects hypothesis, the authors found that the parasite P. falciparum had no structure at all, whereas the vector A. gambiae still showed some structuring between villages (Prugnolle et al. 2008). But this study also lacked data on the other (human) host. Yet these type of studies will be required more to discriminate between the hypotheses of resembling the most vagile host and the additive effects of both hosts.

\section{Conclusion}

The parasite's genetic structure was clearly not a mere copy of that of one of its hosts, nor an intermediate of the two. We thus found no support for the hypothesis that a parasite's population structure resembles that of its most vagile host species. On the other hand, the observations would be in concordance with a model where the parasites population structure is determined by the additive effects of both vector and host. Our study system, with a specialized parasite and easily traceable vector is the ideal candidate to find such a pattern and discriminate between these different hypotheses. The next important step would be to develop nuclear (microsatellite) markers for P. melanipherus. These would allow us to have a more quantifiable and comparable measure of population differentiation in the parasite, as well as enable us the detection of any cryptic species. In parallel, mathematical models need to be developed which can create quantifiable predictions on how host and vector dispersals combined create the parasite's pattern of gene flow.

The genetics of N. schmidlii indicated that ample nonreproductive contact exists between European M. schreibersii subpopulations, which demonstrates the utility of using (ecto) parasites as an ecological 'tag' for host behaviour. In contrast, even though $P$. melanipherus is unquestionably 'tagged' onto both of its hosts, its complex message is much harder to read than any single-host parasite system. A future disentanglement of these effects should, however, prove to be some of the more valuable insights into epidemiology of any vectortransmitted disease.

\section{Acknowledgements}

The authors would like to thank Mikael Paillet, Giada Giacomini, Chiara Varoli, Pamela Priori, Xavier Puig-Montserrat, Arnau Sardà Forcadell, Enric Fàbregas and Beat Goldstein for their help collecting samples. Nadir Alvarez, Karen McCoy, Alan Brelsford, Ricardo Kanitz and Jessica Purcell provided helpful advice on the analyses. Jessica Purcell also proofread an earlier version of this manuscript. Our acknowledgements also go to three anonymous reviewers for their suggestions to improve this manuscript. This study was made possible by financial support of Fondation Herbette as well as grant 31003A_120479 and 31003A_138187 from the Swiss National Science Foundation. 


\section{References}

Bensch S, Stjernman M, Hasselquist D et al. (2000) Host specificity in avian blood parasites: a study of Plasmodium and Haemoproteus mitochondrial DNA amplified from birds. Proceedings of the Royal Society B-Biological Sciences, 267, 15831589.

Bensch S, Perez-Tris J, Waldenstrom J, Hellgren O (2004) Linkage between nuclear and mitochondrial DNA sequences in avian malaria parasites: multiple cases of cryptic speciation? Evolution, 58, 1617-1621.

Blasco-Costa I, Poulin R (2013) Host traits explain the genetic structure of parasites: a meta-analysis. Parasitology, 140, 1316-1322.

Bruyndonckx N, Dubey S, Ruedi M, Christe P (2009) Molecular cophylogenetic relationships between European bats and their ectoparasitic mites (Acari, Spinturnicidae). Molecular Phylogenetics and Evolution, 51, 227-237.

Bruyndonckx N, Biollaz F, Dubey S, Goudet J, Christe P (2010) Mites as biological tags of their hosts. Molecular Ecology, 19, 2770-2778

Clement M, Posada D, Crandall KA (2000) TCS: a computer program to estimate gene genealogies. Molecular Ecology, 9, $1657-1659$

Curtis VA (2014) Infection-avoidance behaviour in humans and other animals. Trends in Immunology, 35, 457-464.

Dick CW, Patterson BD (2008) An excess of males: skewed sex ratios in bat flies (Diptera: Streblidae). Evolutionary Ecology, 22, 757-769.

Dray S, Dufour AB (2007) The ade4 package: implementing the duality diagram for ecologists. Journal of Statistical Software, 22, 1-20.

Duval L, Mejean C, Maganga GD et al. (2012) The chiropteran haemosporidian Polychromophilus melanipherus: a worldwide species complex restricted to the family Miniopteridae. Infection, Genetics and Evolution, 12, 1558-1566.

Earl DA, Vonholdt BM (2012) STRUCTURE HARVESTER: a website and program for visualizing STRUCTURE output and implementing the Evanno method. Conservation Genetics Resources, 4, 359-361.

Estrada-Peña A, Serra-Cobo J (1991) The Acarinia and Nycteribidia zones of Miniopterus schreibersii Kuhl (Mammalia: Chiroptera) in the northeast of Spain. Folia Parasitologica, 38, 345-354.

Evanno G, Regnaut S, Goudet J (2005) Detecting the number of clusters of individuals using the software STRUCTURE: a simulation study. Molecular Ecology, 14, 2611-2620.

Excoffier L, Smouse PE, Quattro JM (1992) Analysis of molecular variance inferred from metric distances among DNA haplotypes-application to human mitochondrial-DNA restriction data. Genetics, 131, 479-491.

Excoffier L, Laval G, Schneider S (2005) Arlequin (version 3.0): an integrated software package for population genetics data analysis. Evolutionary Bioinformatics, 1, 47-50.

Falush D, Stephens M, Pritchard JK (2003) Inference of population structure using multilocus genotype data: linked loci and correlated allele frequencies. Genetics, 164, 1567-1587.

Fumagalli L, Taberlet P, Favre L, Hausser J (1996) Origin and evolution of homologous repeated sequences in the mitochondrial DNA control region of shrews. Molecular Biology and Evolution, 13, 31-46.
Gardner RA, Molyneux DH, Stebbings RE (1987) Studies on the prevalence of Hematozoa of British bats. Mammal Review, 17, 75-80.

Garnham PCC (1966) Malaria Parasites and Other Haemosporidia, 1st edn. Blackwell Scientific Publications, Oxford.

Gomez-Diaz E, Boulinier T, Sertour N et al. (2011) Genetic structure of marine Borrelia garinii and population admixture with the terrestrial cycle of Lyme borreliosis. Environmental Microbiology, 13, 2453-2467.

Goudet J (2001) FSTAT, a program to estimate and test gene diversities and fixation indices (version 2.9.3). Available from http://www.unil.ch/izea/softwares/fstat.html. Updated from Goudet (1995).

Goudet J (2005) HIERFSTAT, a package for R to compute and test hierarchical F-statistics. Molecular Ecology Notes, 5, 184186

Jarne P, Théron A (2001) Genetic structure in natural populations of flukes and snails: a practical approach and review. Parasitology, 123, S27-S40.

Jost L (2008) G(ST) and its relatives do not measure differentiation. Molecular Ecology, 17, 4015-4026.

Kimura M (1980) A simple method for estimating evolutionary rates of base substitutions through comparative studies of nucleotide-sequences. Journal of Molecular Evolution, 16, 111120.

Lanza B (1999) I parassiti dei pipistrelli (Mammalia, Chiroptera) della fauna italiana. Museo Regionale di Scienze Naturali Torino, Turin.

Levin II, Parker PG (2013) Comparative host-parasite population genetic structures: obligate fly ectoparasites on Galapagos seabirds. Parasitology, 140, 1061-1069.

Louhi KR, Karvonen A, Rellstab C, Jokela J (2010) Is the population genetic structure of complex life cycle parasites determined by the geographic range of the most motile host? Infection, Genetics and Evolution, 10, 1271-1277.

Lourenço S, Palmeirim JM (2008) Which factors regulate the reproduction of ectoparasites of temperate-zone cave-dwelling bats? Parasitology Research, 104, 127-134.

Marshall AG (1970) The life cycle of Basilia hispida Theodor 1967 (Diptera: Nycteribiidae) in Malaysia. Parasitology, 61, 118.

McCoy KD, Boulinier T, Tirard C (2005a) Comparative hostparasite population structures: disentangling prospecting and dispersal in the black-legged kittiwake Rissa tridactyla. Molecular Ecology, 14, 2825-2838.

McCoy KD, Chapuis E, Tirard C et al. (2005b) Recurrent evolution of host-specialized races in a globally distributed parasite. Proceedings of the Royal Society B-Biological Sciences, 272, 2389-2395.

Megali A, Yannic G, Christe P (2011) Disease in the dark: molecular characterization of Polychromophilus murinus in temperate zone bats revealed a worldwide distribution of this malaria-like disease. Molecular Ecology, 20, 1039-1048.

Miller-Butterworth CM, Jacobs DS, Harley EH (2002) Isolation and characterization of highly polymorphic microsatellite loci in Schreibers' long-fingered bat, Miniopterus schreibersii (Chiroptera: Vespertilionidae). Molecular Ecology Notes, 2, 139-141.

Nieberding CM, Olivieri I (2007) Parasites: proxies for host genealogy and ecology? Trends in Ecology \& Evolution, 22, 156-165. 
Paradis E (2010) pegas: an R package for population genetics with an integrated-modular approach. Bioinformatics, 26, 419420.

Petersen FT, Meier R, Kutty SN, Wiegmann BM (2007) The phylogeny and evolution of host choice in the Hippoboscoidea (Diptera) as reconstructed using four molecular markers. Molecular Phylogenetics and Evolution, 45, 111-122.

Pritchard JK, Stephens M, Donnelly P (2000) Inference of population structure using multilocus genotype data. Genetics, 155, 945-959.

Prugnolle F, Théron A, Pointier JP et al. (2005) Dispersal in a parasitic worm and its two hosts: consequence for local adaptation. Evolution, 59, 296-303.

Prugnolle F, Durand P, Jacob K et al. (2008) A comparison of Anopheles gambiae and Plasmodium falciparum genetic structure over space and time. Microbes and Infection, 10, 269275.

R Core Team (2012) R: A Language and Environment for Statistical Computing. R Foundation for Statistical Computing, Vienna, Austria.

Ramos Pereira MJ, Salgueiro P, Rodrigues L, Coelho MM, Palmeirim JM (2009) Population structure of a cave-dwelling bat, Miniopterus schreibersii: does it reflect history and social organization? Journal of Heredity, 100, 533-544.

Reckardt K, Kerth G (2006) The reproductive success of the parasitic bat fly Basilia nana (Diptera: Nycteribiidae) is affected by the low roost fidelity of its host, the Bechstein's bat (Myotis bechsteinii). Parasitology Research, 98, 237-243.

Rodrigues L, Palmeirim JM (2008) Migratory behaviour of the Schreiber's bat: when, where and why do cave bats migrate in a Mediterranean region? Journal of Zoology, 274, 116-125.

Rodrigues L, Pereira MJR, Rainho A, Palmeirim JM (2010) Behavioural determinants of gene flow in the bat Miniopterus schreibersii. Behavioral Ecology and Sociobiology, 64, 835-843.

Rousset F (1997) Genetic differentiation and estimation of gene flow from F-statistics under isolation by distance. Genetics, 145, 1219-1228.

Ryberg O (1947) Studies on Bats and Bat Parasites. Bokförlaget Svensk Natur, Stockholm.

Tamura K, Peterson D, Peterson N et al. (2011) MEGA5: molecular evolutionary genetics analysis using maximum likelihood, evolutionary distance, and maximum parsimony methods. Molecular Biology and Evolution, 28, 2731-2739.

Theodor O (1957) Parasitic adaptation and host-parasite specificity in the pupiparous Diptera. In: First Symposium on Host Specificity among Parasites of Vertebrates (ed. Mayr E), pp. 5063. Union Internationale des Sciences Biologiques Série B, Neuchâtel.

Theodor O (1967) An Illustrated Catalogue of the Rothschild Collection of Nycteribiidae. British Museum (Natural History), London.

Van Oosterhout C, Hutchinson WF, Wills DPM, Shipley P (2004) MICRO-CHECKER: software for identifying and correcting genotyping errors in microsatellite data. Molecular Ecology Notes, 4, 535-538

Waite JL, Henry AR, Adler FR, Clayton DH (2012) Sex-specific effects of an avian malaria parasite on an insect vector: support for the resource limitation hypothesis. Ecology, 93, 24482455.

Witsenburg F, Schneider F, Christe P (2014) Signs of a vector's adaptive choice: on the evasion of infectious hosts and parasite-induced mortality. Oikos, online early view. doi:10.1111/oik.01785.

Wood R, Weyeneth N, Appleton B (2011) Development and characterisation of 20 microsatellite loci isolated from the large bent-wing bat, Miniopterus schreibersii (Chiroptera: Miniopteridae) and their cross-taxa utility in the family Miniopteridae. Molecular Ecology Resources, 11, 675-685.

Wright S (1951) The genetical structure of populations. Annals of Eugenics, 15, 323-354.

F.W., N.S. and P.C. conceived and designed the study. L.C., A.L.B., J.P., I.P., D.S. and M.S. organized local permissions, material and sampling schemes. F.W., L.C., A.L.B., J.P., I.P., D.S., M.S. and L.D. collected the samples. L.C. and F.W. tested and designed molecular tools, performed molecular analyses, scored alleles and haplotypes and performed a preliminary data analysis. F.W. performed the final data analysis and wrote the manuscript. J.G. and P.C. contributed to the data analysis, interpretation of the results and writing of the manuscript.

\section{Data accessibility}

DNA sequences of the new Nycteribia schmidlii microsatellites: GenBank Accession nos KJ189108-KJ189117. Mitochondrial haplotypes of Polychromophilus melanipherus, Miniopterus schreibersii and N. schmidlii: GenBank Accession nos KJ131270-KJ131277, KJ131278-KJ131380 and KJ131381-KJ131412 respectively. All DNA sequence alignments: Dryad doi: 10.5061/dryad.2m1p0. Microsatellite genotypes, sex, host id. and sampling locations of M. schreibersii and N. schmidlii: Dryad doi: 10.5061/ dryad.2m1p0.

\section{Supporting information}

Additional supporting information may be found in the online version of this article.

Fig. S1 Isolation-by-distance patterns for M. schreibersii (A) and N. schmidlii (B) based on microsatellite data. (C) No correlation between the vector and host population differentiation patterns.

Fig. S2 Structure assignment plots for Miniopterius schreibersii based on 11 microsatellites.

Fig. S3 Infection rates of $M$. schreibersii with the haemosporidian parasite $P$. melanipherus plotted per sampling site.

Fig. S4 Isolation-by-distance patterns for M. schreibersii (A), N. schmidlii (B) and P. melanipherus (C) based on mitochondrial sequence data.

Table S1 M. schreibersii microsatellites markers used in this study. 
940 F. WITSENBURG ET AL.

Table S2 Thermal profiles used for the amplification reactions in this study.

Table S3 Reagents and their respective concentrations used for the amplification reactions in this study.

Table S4 Newly developed microsatellite markers for N. schmidlii.

Table S5 Molecular diversity indices based on microsatellite data for M. schreibersii and N. schmidlii.

Table S6 Basic population genetic statistics of $M$. schreibersii and N. schmidlii, averaged over 11, 8 microsatellites respectively.
Table S7 Gene diversity measures of the parasite P. melanipherus, the host M. schreibersii and vector N. schmidlii, based on their mtDNA fragments.

Table S8 Pairwise genetic distances between sampling sites of M. schreibersii.

Table S9 Pairwise genetic distances between sampling sites of N. schmidlii.

Table S10 Pairwise genetic distances between sampling sites of P. melanipherus. 